See discussions, stats, and author profiles for this publication at: https://www.researchgate.net/publication/280971212

\title{
Energy Efficient Bioethanol Purification by Heat Pump Assisted Extractive Distillation
}

Conference Paper in Computer Aided Chemical Engineering · May 2015

DOI: $10.1016 / 8978-0-444-63577-8.50063-2$

CITATIONS

3 authors:

Anton Alexandru Kiss

The University of Manchester

217 PUBLICATIONS 3,809 CITATIONS

SEE PROFILE

Costin Sorin Bildea

Polytechnic University of Bucharest

182 PUBLICATIONS 1,381 CITATIONS

SEE PROFILE

Some of the authors of this publication are also working on these related projects:

PRISM - Towards knowledge based processing systems View project

Design and Control of Chemical Plants Coupling Exothermic and Endothermic Reactions View project
225

Hao Luo

Technical University of Denmark

6 PUBLICATIONS 77 CITATIONS

SEE PROFILE 
Krist V. Gernaey, Jakob K. Huusom and Rafiqul Gani (Eds.), 12th International Symposium on Process Systems Engineering and 25th European Symposium on Computer Aided Process Engineering. 31 May - 4 June 2015, Copenhagen, Denmark (c) 2015 Elsevier B.V. All rights reserved.

\title{
Energy Efficient Bioethanol Purification by Heat Pump Assisted Extractive Distillation
}

\author{
Anton A. Kiss ${ }^{\mathrm{a}, \mathrm{b}^{*}}$, Hao Luo ${ }^{\mathrm{c}, \mathrm{d}}$, Costin Sorin Bildea $^{\mathrm{e}}$ \\ ${ }^{a}$ AkzoNobel Research, Development \& Innovation, Process Technology SRG, \\ Zutphenseweg 10,7418 AJ Deventer, The Netherlands.Tony.Kiss@akzonobel.com \\ ${ }^{b}$ Sustainable Process Technology Group, Faculty of Science and Technology, \\ University of Twente, PO Box 217, 7500 AE, Enschede, The Netherlands \\ ${ }^{c}$ State Key Laboratory of Multi-Phase Complex Systems, Institute of Process \\ Engineering, Chinese Academy of Sciences, Beijing 100190, China \\ ${ }^{d}$ Sino-Danish Center for Education and Research, Beijing 100190, China \\ eUniversity "Politehnica” of Bucharest, Polizu 1-7, 011061 Bucharest, Romania
}

\begin{abstract}
The purification of bioethanol fuel requires an energy demanding separation process to concentrate the diluted streams obtained in the fermentation stage and to overcome the azeotropic behaviour of ethanol-water mixture. The classic separation sequence consists of three distillation columns that carry out several energy demanding tasks: preconcentration of ethanol, extractive distillation and solvent recovery.

To solve this problem, we propose a novel heat pump assisted extractive distillation process taking place in a dividing-wall column (DWC). In this configuration, the ethanol top vapour stream of the extractive DWC is recompressed from atmospheric pressure to over 3.1 bar (thus to a higher temperature) and used to drive the side reboiler of the DWC, which is responsible for the water vaporization. The results show that the specific energy requirements drop from $2.07 \mathrm{kWh} / \mathrm{kg}$ (classic sequence) to only 1.24 $\mathrm{kWh} / \mathrm{kg}$ ethanol (VRC assisted extractive DWC), thus energy savings of over $40 \%$ and $24 \%$ reduced total annual costs are possible, as compared to the conventional process.
\end{abstract}

Keywords: extractive distillation; dividing-wall column; heat pump; energy savings

\section{Introduction}

The industrial production of bioethanol relies on various routes: corn-to-ethanol, sugarcane-to-ethanol, basic and integrated lignocellulosic biomass-to-ethanol. In all cases, the raw materials undergo several pre-treatment steps before entering the fermentation stage where bioethanol is produced. A common feature is the production of diluted bioethanol (typically 5-12 \%wt ethanol) that is further concentrated to reach the requirements of the international bioethanol standards (Vane, 2008). To reach the purity targets (max. $0.2 \%$ vol water), an energy demanding separation is needed in practice, in order to overcome the presence of the binary azeotrope ethanol-water (95.63 $\%$ wt ethanol). The separation is typically carried out by distillation, the first step being a pre-concentration distillation column (PDC) that increases the ethanol content from 5$12 \%$ wt up to 91-94\%wt (Frolkova and Raeva, 2010). The second step consists of the ethanol dehydration, up to concentrations exceeding the azeotropic composition. Quite a number of separation alternatives are available as described in the open literature: pervaporation, adsorption, pressure-swing distillation (PSD), extractive distillation 
(ED), azeotropic distillation (AD), and hybrid methods combining these options (Vane, 2008; Frolkova and Raeva, 2010). Among them, extractive distillation (ED) is still the option of choice in case of large scale production of bioethanol fuel (Kiss and Ignat, 2012). Typically, ED is performed in a sequence of two columns, one being the extractive distillation column (EDC) which separates ethanol, while the other one is the solvent recovery column (SRC) that recovers the mass separating agent (MSA) which is recycled back in the process. Further improvements to the distillation process were proposed, with the aim to increase the energy efficiency of bioethanol purification: e.g. ED process optimization (Kiss and Ignat, 2013); thermally coupled distillation columns; azeotropic and extractive dividing-wall columns (Kiss, 2013).

This work proposes a novel heat pump assisted ED process that efficiently combines vapour recompression (VRC) heat pump with dividing-wall column technology, and allows a major reduction of the energy requirements. A $100 \mathrm{ktpy}$ plant is considered, processing a feed with $10 \%$ wt ethanol, by extractive distillation using ethylene glycol as solvent. Simulations were carried out in Aspen Plus, using NRTL model, and for a fair process comparison the same approach was used as in previous studies (Kiss, 2013).

\section{Problem statement}

For its use as biofuel or additive, bioethanol must have a purity of min. 99-99.8 \% wt but this can be reached only by paying a large energy penalty. Although most of the water present in the diluted ethanol/water mixture (5-12\%wt) from the fermentation step can removed by ordinary distillation, the ethanol purity is limited to max. $95.6 \%$ wt due to the presence of its binary azeotrope with water. Using process optimization and advanced distillation technologies, the energy requirements were reduced by $20 \%$ from about 2.5 down to almost $2 \mathrm{kWh} / \mathrm{kg}$ bioethanol (Kiss, 2013). However, to put this into perspective, the energy needed for purification is still considerably high, especially when taking into account the energy content of the ethanol fuel $(7.45 \mathrm{kWh} / \mathrm{kg}$ fuel).

To solve this issue we integrate VRC technology with an extractive dividing-wall column (E-DWC) capable of purifying bioethanol in a single distillation unit. As shown later, this integration unit allows significant energy savings, less $\mathrm{CO}_{2}$ emissions and a reduced footprint, as compared to the conventional separation process.

\section{Results and discussion}

\subsection{Classic extractive distillation process}

Figure 1 presents the process flowsheet, including the mass and energy balance along with the key process parameters of the classic ethanol purification sequence based on extractive distillation (Kiss and Ignat, 2013).

The process consists of three distillation columns: pre-concentration distillation (PDC), extractive distillation (EDC) and solvent recovery (SRC). The PDC unit separates water as bottom stream and a near-azeotropic composition mixture as distillate (91\%wt ethanol being the optimal value as reported by Kiss and Ignat, 2013). The second column (EDC) uses ethylene glycol as solvent that is added at a solvent-to-ethanol ratio of $1.25 \mathrm{~mol} / \mathrm{mol}$, on a stage above the feed stage of the near azeotropic ethanol-water mixture. The presence of the solvent changes the relative volatility of ethanol-water such that their separation becomes possible. High purity ethanol is collected as top distillate product of the EDC, while the bottom stream contains solvent and water. The third column (SRC) in the sequence separates the remaining water as distillate and completely recovers the solvent as bottom product, which is cooled in a feed-effluent heat exchanger (FEHE) and then recycled back to the extractive distillation column. 


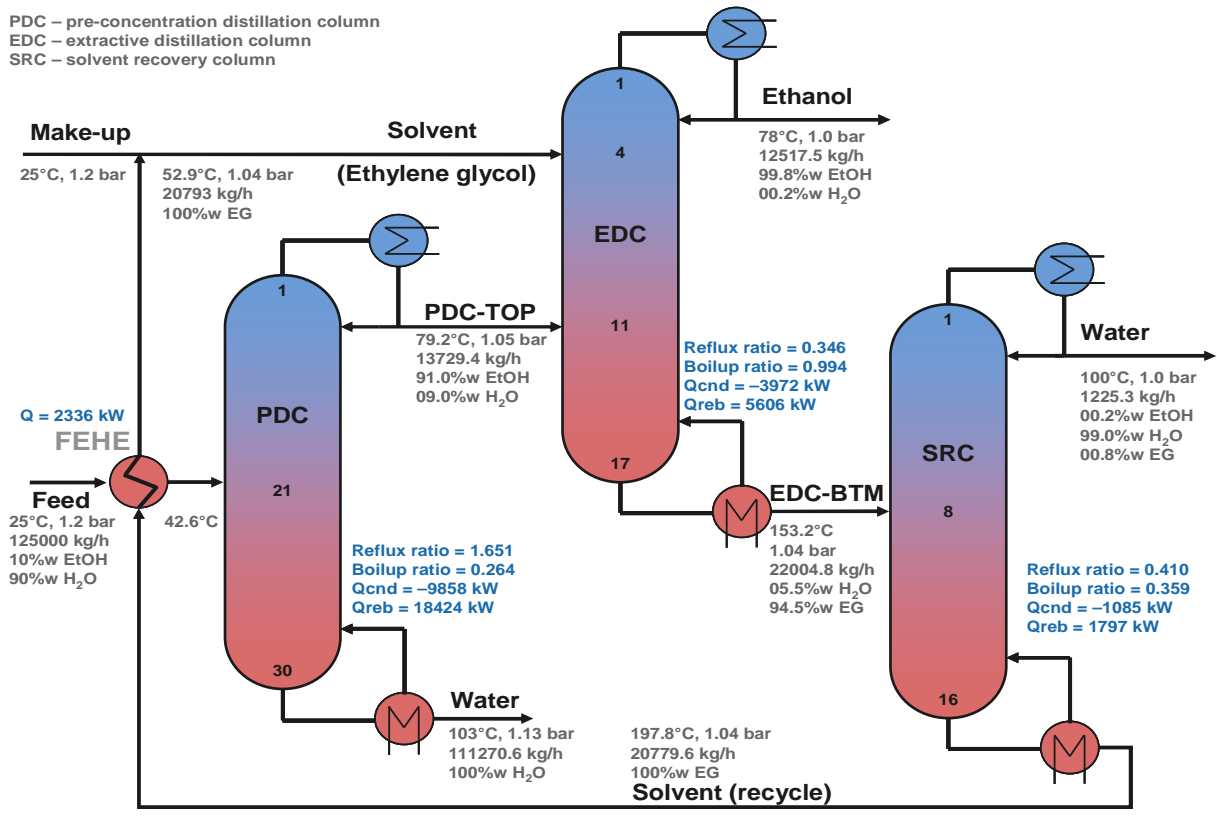

Figure 1. Flowsheet including key process parameters of the classic bioethanol separation by extractive distillation (the numbers on columns indicate the top, bottom and feed stage)

\subsection{Vapour recompression assisted extractive DWC}

Heat pump assisted distillation can be applied to classic columns (Kiss et al., 2012) but only to a lesser extent to dividing-wall columns (Chew et al., 2014) as the temperature span across the DWC is larger. Kiss et al. (2012) provided a useful scheme for the quick selection of appropriate heat pumps, while Plesu et al. (2014) introduced a criterion depending on the Carnot efficiency to decide whether a heat pump is worth considering:

$Q / W=1 / \eta=T_{\mathrm{c}} /\left(T_{\mathrm{r}}-T_{\mathrm{c}}\right)>10$

where $Q$ is the reboiler duty, $W$ the work provided, while $\eta$ is the Carnot efficiency, $T_{\mathrm{r}}$ the reboiler temperature and $T_{\mathrm{c}}$ the condenser temperature. When the $Q / W$ ratio exceeds 10 , then a heat pump should be considered, while when the ratio is lower than 5 then using a heat pump will not bring any benefits (Plesu et al., 2014). In case of the ethanol separation, the ratio $Q / W$ is $16\left(T_{\mathrm{r}}=100{ }^{\circ} \mathrm{C}, T_{\mathrm{c}}=78^{\circ} \mathrm{C}\right)$ which is clearly favourable.

Notably, the use of a DWC was already explored as an attractive process alternative for bioethanol purification (Kiss and Ignat, 2012). Hereby we go further, by combining the E-DWC technology with vapour recompression (VRC) in order to increase more the energy efficiency. Figure 2 presents the flowsheet of the novel process based on VRC assisted extractive distillation in a DWC. For the reader's convenience, the mass and energy balance, as well as the key process parameters are also provided.

The feed side (prefractionator) acts as the PDC unit of the classic sequence. Water is removed as liquid side stream, but an additional side reboiler is needed in order to return the required amount of water vapours to the column. The liquid feed stream is fed on top of the prefractionator side, thus serving as a reflux to the PDC section. The vapour leaving the feed side of the E-DWC is enriched in ethanol. Solvent is added at the top of the E-DWC, this section acting in fact as the EDC unit of the conventional sequence. 


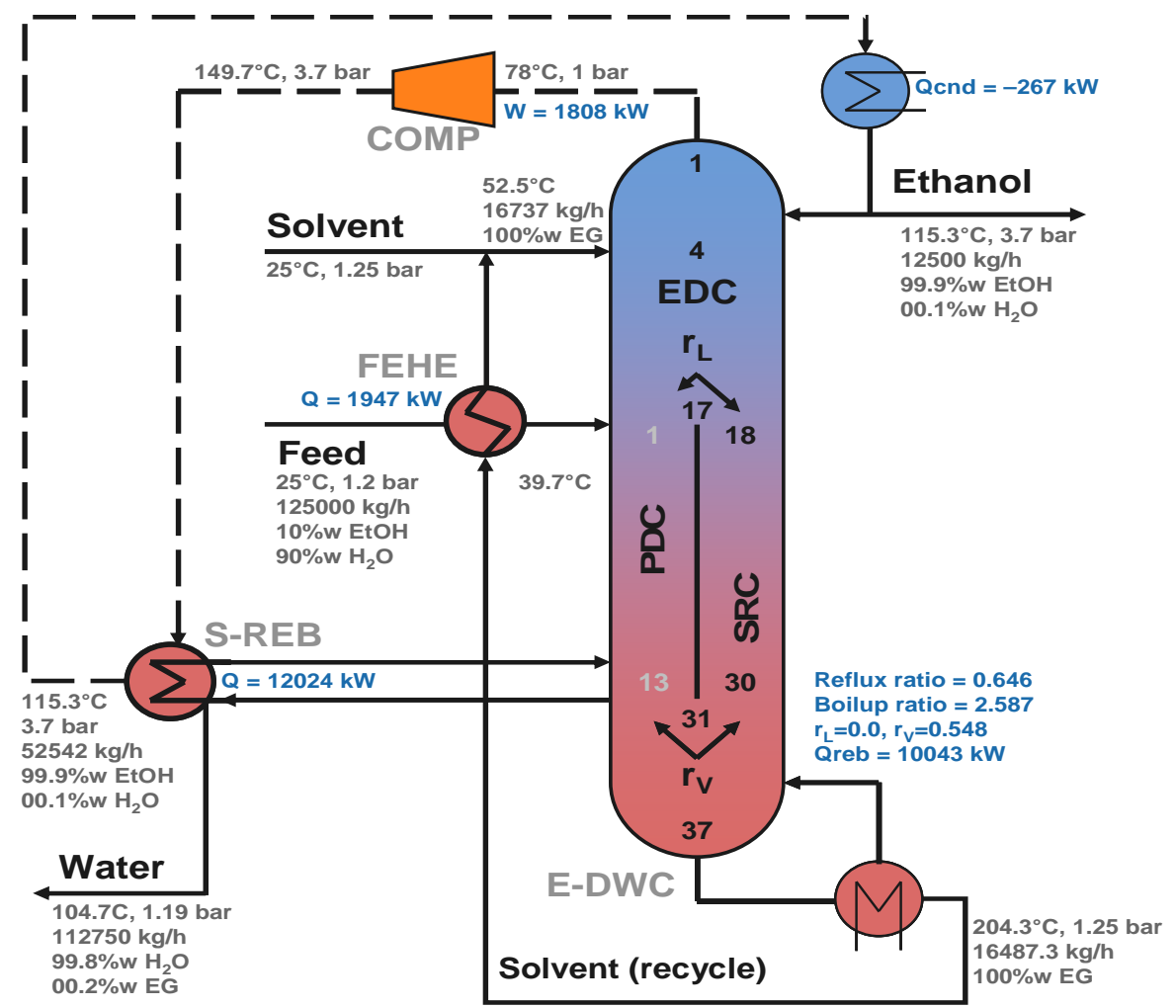

Figure 2. Flowsheet including the mass balance and key parameters of the novel process for bioethanol purification based on VRC assisted extractive distillation in a DWC.

Ethanol is separated here as high purity vapour distillate that is compressed to a higher pressure and temperature level, and then used to drive the side reboiler of the column and being eventually condensed and removed as main product. The liquid flowing down the top section (EDC) is collected and distributed only to the (SRC) side opposite to the feed side and further down the bottom of the E-DWC. This complete redistribution of the liquid flow is required in order to avoid the presence and loss of solvent on the feed side (PDC section). In the SRC section, the solvent is separated as bottom product then cooled in a heed-effluent heat exchanger (FEHE) and recycled to the E-DWC unit. The vapour from the bottom of the E-DWC to the lower part of the dividing-wall is not sufficient for the PDC section, thus the need for an additional side reboiler - which can be driven by a heat pump (VRC in this case). In spite of the high degree of integration of E-DWC, the controllability of such systems is satisfactory (Tututi-Avila et al., 2014).

\subsection{Sensitivity analysis}

Integrating a vapour recompression heat pump with an extractive DWC requires the setting of the appropriate discharge pressure from the compressor. Figure 3 shows the dependence of the compressor duty and the inverse of the log-mean temperature difference (LMTD) on the discharge pressure of the compressor involved by the VRC system. In terms of reducing the VRC costs, the compressor duty should be as low as possible (smaller and cheaper compressor), while the LMTD should be as high as possible (larger driving force and smaller heat exchanger). 


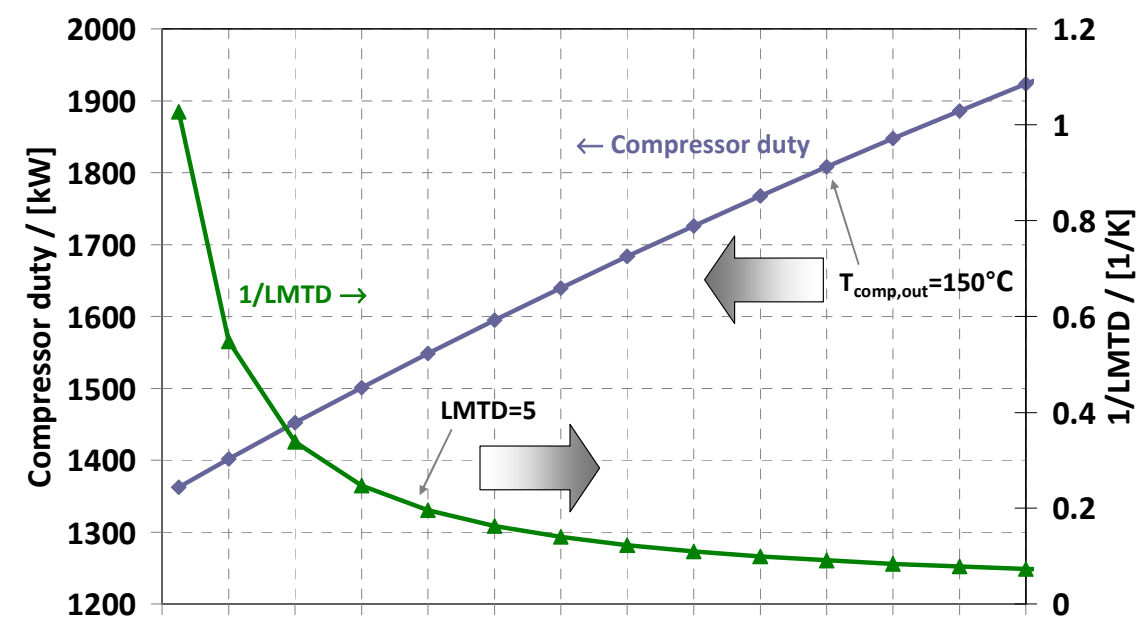

$\begin{array}{llllllllllllll}2.7 & 2.8 & 2.9 & 3.0 & 3.1 & 3.2 & 3.3 & 3.4 & 3.5 & 3.6 & 3.7 & 3.8 & 3.9 & 4.0\end{array}$ Pressure / [bar]

Figure 3. Dependence of compressor duty and the log-mean temperature difference (LMTD) in the side-reboiler, on the discharge pressure of the compressor (VRC system)

However, practical limitations are imposed and these reduce the available range for the discharge pressure to 3.1-3.7 bar. For the heat exchanger (side reboiler of E-DWC) which is part of the VRC loop, the LMTD must exceed $5 \mathrm{~K}$ to obtain a reasonable sized and inexpensive side-reboiler - as the heat exchange area (A) depends proportionally with the inverse of LMTD (e.g. $\mathrm{A}=\mathrm{Q} / \mathrm{U} \times 1 / \mathrm{LMTD})$, when considering a constant heat duty $(\mathrm{Q})$ and heat transfer coefficient (U). Similarly, the compressor is also limited not only by the compression ratio (typically up to $2.5-4.0$ ), but mainly by the discharge temperature (DCT) which should not exceed $150^{\circ} \mathrm{C}$ for safety reasons - at higher temperatures the system may fail from worn rings, acid formations and oil breakdown.

\section{Economic evaluation}

The total investment costs (TIC), total operating costs (TOC) and total annual costs (TAC) were calculated according to the procedure described in our previous work (Kiss and Ignat, 2013). The equipment costs are estimated using correlations, based on the Marshall \& Swift equipment cost index $(M \& S=1468.6$ in 2012). A price of 600 US $\$ / \mathrm{m}^{2}$ was used for the sieve trays cost calculations. For the TAC calculations, a plant lifetime of 10 years was used. Furthermore, the following costs were considered for different types of utilities: $0.03 \$ /$ cooling water and 15,17 and $20 \$ /$ steam of low, medium and high pressure, respectively.

Table 1 provides more details about the rest of key performance indicators. Due to the use of a compressor and a larger side-reboiler required by the VRC system, the total investment cost of the proposed VRC E-DWC process is about $29 \%$ higher than for the classical process, but this is largely compensated by the significant energy savings, which exceed $60 \%$ at a direct comparison. When taking into account the inefficiencies in power generation - e.g. by considering that the ratio of heat to electrical $\mathrm{kW}$ is about 3 , then the equivalent energy requirements are $0.805+3 \times 0.145=1.24 \mathrm{kWh} / \mathrm{kg}$ ethanol - the real savings in primary energy become $40 \%$ which is still a remarkable figure. 
Table 1. Process performance comparison (*E-DWC as reported in Kiss and Ignat, 2012)

\begin{tabular}{lccc}
\hline Key performance indicator & Conventional & E-DWC & VRC E-DWC \\
\hline Total investment costs, TIC $(\mathrm{k} \$)$ & 3462 & 3626 & 4477 \\
Total operating costs, TOC $(\mathrm{k} \$ / \mathrm{yr})$ & 5784 & 5355 & 4221 \\
Total annual costs, TAC $(\mathrm{k} \$ / \mathrm{yr})$ & 6130 & 5718 & 4668 \\
$\mathrm{CO}_{2}$ emissions $(\mathrm{kg} \mathrm{CO} /$ / ton product) & 288.94 & 288.31 & 173.04 \\
Thermal energy use $(\mathrm{kWh} / \mathrm{kg}$ product) & 2.07 & 2.07 & 0.80 \\
Electrical energy use $(\mathrm{kWh} / \mathrm{kg}$ product) & $\mathrm{n} / \mathrm{a}$ & $\mathrm{n} / \mathrm{a}$ & 0.14 \\
Equivalent energy requirements $(\mathrm{kWh} / \mathrm{kg})$ & 2.07 & 2.07 & 1.24 \\
\hline
\end{tabular}

Considering both the investment and operating costs, the total annual cost (TAC) is reduced by about $24 \%$, while the $\mathrm{CO} 2$ emissions - which are closely linked to the primary energy requirements - are reduced by $40 \%$ (or even $60 \%$ when the electricity for the compressor comes from renewable sources).

\section{Conclusions}

The novel heat pump assisted extractive distillation process proposed here is based on an efficient combination of vapour recompression (VRC) and DWC technology. In this configuration, the ethanol top vapour stream of the extractive DWC is recompressed from atmospheric pressure to 3.1-3.7 bar thus to a higher temperature, and used to drive the side reboiler responsible for water vaporization. The results show that the specific energy requirements drop from $2.07 \mathrm{kWh} / \mathrm{kg}$ (classic sequence) to only $1.24 \mathrm{kWh} / \mathrm{kg}$ ethanol (VRC assisted extractive DWC) hence energy savings of $40 \%$ being possible. Considering the requirements for a compressor and use of electricity in case of the heat pump assisted alternative, about $24 \%$ decrease of the total annual costs is possible for the novel process (in spite of the $29 \%$ increase of total investment costs), as compared to the classic extractive distillation.

\section{References}

J. M. Chew, C.C.S. Reddy, G.P. Rangaiah, 2014, Improving energy efficiency of dividing-wall columns using heat pumps, Organic Rankine Cycle and Kalina Cycle. Chemical Engineering and Processing, 76, 45-59.

A. K. Frolkova, V. M. Raeva, 2010, Bioethanol dehydration: State of the art. Theoretical Foundations of Chemical Engineering, 44, 545-556.

A. A. Kiss, 2013, Novel applications of dividing-wall column technology to biofuel production processes. Journal of Chemical Technology and Biotechnology, 88, 1387-1404.

A. A. Kiss, S. J. Flores Landaeta, C. A. Infante Ferreira, 2012, Towards energy efficient distillation technologies - Making the right choice. Energy, 47, 531-542.

A. A. Kiss, R. M. Ignat, 2012, Innovative single step bioethanol dehydration in an extractive dividing-wall column. Separation \& Purification Technology, 98, 290-297.

A. A. Kiss, R. M. Ignat, 2013, Optimal economic design of a bioethanol dehydration process by extractive distillation. Energy Technology, 1, 166-170.

V. Plesu, A. E. Bonet Ruiz, J. Bonet, J. Llorens, 2014, Simple equation for suitability of heat pump use in distillation, Computer Aided Chemical Engineering, 33, 1327-1332.

S. Tututi-Avila, A. Jimenez-Gutierrez, J. Hahn, 2014, Control analysis of an extractive dividingwall column used for ethanol dehydration. Chemical Engineering and Processing, 82, 88-100.

L. M. Vane, 2008, Separation technologies for the recovery and dehydration of alcohols from fermentation broths, Biofuels, Bioproducts and Biorefining, 2, 553-588. 